\title{
Extraction of baryonia from the lightest antiprotonic atoms
}

\author{
B. Loiseau \\ Sorbonne Universités, Université Pierre et Marie Curie, \\ Sorbonne Paris Cité, Université Paris Diderot, et IN2P3-CNRS, UMR 7585, \\ Laboratoire de Physique Nucléaire et de Hautes Énergies, 4 place Jussieu, 75252 Paris, France
}

S. Wycech

National Centre for Nuclear Research, Pasteura 7, 02-093 Warsaw, Poland

(Dated: October 14, 2020)

\begin{abstract}
Antiprotonic hydrogen and helium atoms are analyzed. Level shifts and width are expressed in terms of $\bar{p}$-nucleon subthreshold scattering lengths and volumes. Experimental data are compared to results obtained from the 2009 version of the Paris $N \bar{N}$ interaction potential. Comparison with the 1999 version is also made. Effects of $N \bar{N}$ quasibound states are discussed. Atomic $2 \mathrm{P}$ hyperfine structure is calculated for antiprotonic deuterium and the significance of new measurements is indicated.

PACS numbers: 13.75.-n, 36.10-k, 25.80.-e, 25.40.Ve
\end{abstract}

\section{INTRODUCTION}

The lightest hadronic atoms offer a chance to test hadron-nucleon scattering amplitudes just below the thresholds. This energy region is of special interest in cases of bound or quasibound states in the hadron nucleon systems. Two cases of current interest the $\bar{p}$ and $K^{-}$atoms are similar in this respect. Here we discuss the case of the antiproton. Nucleon-antinucleon bound states (baryonia) have been searched for since the beginnings of the LEAR era at CERN. Nothing definite was found, apparently because of two factors. First, these states are broad because fast annihilation processes and experiments are confronted with heavy backgrounds. Second, the exclusion principle is not operative and a large number of partial waves may be formed in the $N \bar{N}$ systems.

High selectivity is needed to detect a clear signal. One way to reach selected states is a formation reaction. In this way a resonant-like behavior was observed in the decay $J / \psi \rightarrow \gamma p \bar{p}$. The experiment performed by the BES Collaboration [1] found an enhancement in the $\bar{p} p$ close to the threshold energy equal to $1876 \mathrm{MeV}$. With a model it was attributed to a subthreshold peak at invariant mass of $1859 \mathrm{MeV}$. This was confirmed by the BES III Collaboration 2, and the extrapolation has led to a peak at $1832 \mathrm{MeV}$. This may be, but not necessarily is, a confirmation of a quasibound state. The valid proof requires a look into the subthreshold region and this also was achieved by the BES Collaboration [3] in the mesonic decays of $J / \psi$ into $\gamma \pi^{+} \pi^{-} \eta^{\prime}$. A subthreshold enhancement, the $\mathrm{X}(1835)$, was found in this way.

Interpretation, in the $J / \psi \rightarrow \gamma p \bar{p}$ process, of the close to $\bar{p} p$ threshold enhancement in terms of $N \bar{N}$ potentials 4, 5, leads to the conclusion that there exist a broad spin singlet $S$-wave quasibound state. However, the state obtained [5] by Bonn potential is formed in the isospin $I=1$. That generated by Paris potential [6] happens in $I=0$ at the energy $-4.8 \mathrm{MeV}$ with a width of $50 \mathrm{MeV}$, state which we named $N \bar{N}_{S}(1870)$ [7. Paris potential explains the $X(1835) \mathrm{MeV}$ enhancement as a result of an interference of this quasibound $S$-wave state with a background amplitude [7].

Paris 2009 potential generates also a deeply bound, isospin $1 S$-wave state at about -80 MeV binding. This state is far away from the experimentally tested energy regions and may be an artifact of unknown interactions at very short ranges. Nevertheless, it exists in the model and we find that after some modifications it may play a significant role in the antiprotonic-atomic physics.

There is a class of selective experiments which also allow one to test the subthreshold energy region. These are the measurements of atomic levels in very light atoms. The condition for a direct partial wave analysis is the resolution of the fine structure of these levels. So far, such resolution was achieved only in the antiprotonic hydrogen [8, 9] and it is instrumental in fixing the $N \bar{N}$ potential at low energy $[\underline{6}$. Other atoms useful to study baryonia are antiprotonic deuterium and antiprotonic helium, as, in these atoms, the experimental data exist. The advantage of such systems lies in their (relatively) simple nuclear structure and the fact that $\bar{p} N$ interactions happen on bound nucleons of well defined separation energy. In this way one can study the $\bar{p} N$ interaction below the threshold, moreover at different subthreshold energies.

In this work we study the level shifts and widths from $\bar{p} N$ interactions in the lightest atoms (levels): ${ }^{2} \mathrm{H}(2 P)$, ${ }^{3} \mathrm{He}(2 P, 3 D)$ and ${ }^{4} \mathrm{He}(2 P, 3 D)$. The number of states is limited to those characterized by very low atomic-nuclear overlaps. In these states, multiple scattering expansions converge very quickly and double scattering or interactions on two nucleons contribute small fractions of the level shifts. Experiments discussed are fairly old, 9 11, but have been analyzed only in a phenomenological way and never discussed in terms of a well established $\bar{p} N$ potential. 
Understanding of the simplest atoms is important for the PUMA project at CERN aiming at studies of antiproton capture from atomic states formed on unstable nuclei [12. Relative frequency of $\bar{p} p$ and $\bar{p} n$ captures will be measured with the intention to determine neutron haloes in unstable nuclei. These nuclei are likely to contain loosely bound nucleons, thus kinematic conditions may be similar to antiprotonic-deuterium and ${ }^{3}$ He atoms. Knowledge of the level widths structure in these atoms would be helpful for this project. Related experiments were performed on stable nuclei [13. In general the ratio of captures on neutrons and protons is well controlled, but it was found that some irregularities happen for loosely bound protons [14. One hopes that the light atom studies will allow one to pinpoint the partial wave responsible for these effects and will give constraints on the parameters describing heavy antiprotonic atoms.

TABLE I: The ratios of $N(\bar{p} n)$ and $N(\bar{p} p)$ capture rates from atomic states. The second column gives the experimental numbers obtained in radiochemical experiments [14].

\begin{tabular}{lc}
\hline \hline , atom & $N(\bar{p} n) / N(\bar{p} p)$ \\
\hline${ }^{96} \mathrm{Zr}$ & $2.6(3)$ \\
${ }^{124} \mathrm{Sn}$ & $5.0(6)$ \\
\hline${ }^{106} \mathrm{Cd}$ & $0.5(1)$ \\
${ }^{112} \mathrm{Sn}$ & $0.79(14)$ \\
\hline \hline
\end{tabular}

A few selected ratios of $N(\bar{p} n)$ and $N(\bar{p} p)$ capture rates from atomic states are presented in Table Ir. Two normal cases ${ }^{96} \mathrm{Zr}$ and ${ }^{124} \mathrm{Sn}$ indicate neutron haloes. The other two results ${ }^{106} \mathrm{Cd}$ and ${ }^{112} \mathrm{Sn}$ are anomalous and point to proton haloes which cannot be understood by standard nuclear structure models. Only part of the effect is related to a sizable differences $(\sim 3 \mathrm{MeV})$ in the proton and neutron separation energies of the valence nucleons in both of these nuclei. An additional explanation could be related to a fairly narrow $N$ - $\bar{N}$-quasibound state that enhances $\bar{p}$ - $p$ absorptions over $\bar{p}-n$ ones in these nuclei. Existence of a fairly narrow $(\Gamma \leq 10) \mathrm{MeV}$ state in a $P$ wave was predicted in Paris potential models [6] and [15. Its position is, however, not well determined by the scattering data and studies of light atoms might resolve this question.

In conclusion, we argue that there could well be two quasibound isospin $1 N \bar{N}$ states, one in a $S$ wave and another in a $P$ wave. Both are predicted by the recent Paris potential but at incorrect energies. An update of this potential model is required for understanding of the planned CERN and GSI proposals concerning low energy-antiproton research.

This paper is organized as follows. Section II presents a formalism used to calculate the related complex shifts $\Delta E-i \Gamma / 2$. These are expressed in terms of averages of $S$ - and $P$-wave $N \bar{N}$ scattering amplitudes derived from the 2009 [6] and 1999 [15] Paris $N \bar{N}$-interaction potentials and arranged into a sum of multiple scattering series. At this moment, the fine structures in these levels, are, in general, not resolved and the $N \bar{N}$ amplitudes have to be averaged over spin states. Calculated atomic levels using the 2009 and 1999 Paris-potential models are compared to data in section III. This section is also devoted to the extraction of baryonium energies and widths as indicated by the data. Finally we calculate the fine structure of $2 P$ states in deuterium which may be useful to pinpoint properties of the baryonia. Detailed expressions of the equations used in our calculation are presented in an appendix.

\section{FORMALISM}

\section{A. Relation between level shifts and scattering amplitudes}

Experiments which detect the $\mathrm{x}$ rays emitted from hadronic atoms provide energy levels shifted in comparison to the electromagnetic levels by $\Delta E$ from nuclear interactions. The level widths $\Gamma$ frpm antiproton annihilation are also provided in this way. For a given main atomic quantum number $n$ and angular momentum $L$ these complex level shifts are related to the corresponding hadron-nucleus $L$-wave scattering parameter $A_{L}$. The relation is usually obtained by expansion in $A_{L} / B^{L}$ where $B$ is the Bohr radius. The scattering amplitudes are measured experimentally and interpreted in terms of Coulomb plus short-ranged potential solutions. Inner Coulomb corrections are included in $A_{L}$. For $S$ waves such a relation,

$$
\Delta E_{n S}-i \Gamma_{n S} / 2=E_{n S}-\epsilon_{n S}=\frac{2 \pi}{m_{r}}\left|\psi_{n}(0)\right|^{2} A_{0}\left(1+\lambda_{0} A_{0} / B\right)
$$

is known as the Deser-Trueman formula [16, 17. Here, $\psi_{n}(0)$ is the atomic wave function at the origin and $m_{r}$ is the antiproton-nucleus reduced mass. Formula (1) is accurate to a second order in $A_{0} / B$, higher terms in this expansion 
are not needed for small $Z$ nuclei. It was obtained in the non-relativistic limit. Changes are to be introduced in the electromagnetic energy $\epsilon_{n S}$ which is composed of the Bohr's atom energy, $\epsilon_{n}^{0}=-m_{r}(\alpha)^{2} / 2 n^{2}$, corrected for relativity, radiative effects and nuclear polarization, $\alpha$ being the fine structure constant. In the case of antiprotons, the weak singularity of the Dirac wave function has to be smeared over the nuclear density but this brings no noticeable changes.

The Bohr radius is given by $1 /\left(\alpha m_{r}\right)$. In the $1 S$ states one has $\lambda_{0}=3.154$, and with $A_{0} \approx 1 \mathrm{fm}$, the second order term in Eq (1) constitutes a few percent correction. Such corrections are negligible in higher angular momentum states. For these states a simpler linear relation,

$$
\Delta E_{n L}-i \Gamma_{n L} / 2=\epsilon_{n}^{o} \frac{4}{n} \Pi_{i=1}^{L}\left(\frac{1}{i^{2}}-\frac{1}{n^{2}}\right) A_{L} / B^{2 L+1}\left(1+\lambda_{L} A_{L} / B^{2 L+1}\right),
$$

was derived by Lambert [18. The second order correction and inner Coulomb corrections for $N-\bar{N}$ systems may be found in Refs. [19, 20]. For $2 P$ levels $\lambda_{1}=1.866$ and this correction is negligible. Orders of magnitude of the shifts are represented by the coefficients $\Omega_{n L}$ defined as

$$
\Omega_{n L}=\epsilon_{n}^{o} \frac{4}{n} \Pi_{i=1}^{L}\left(\frac{1}{i^{2}}-\frac{1}{n^{2}}\right) / B^{2 L+1},
$$

which, for the simplest atoms, are given in TableII It offers the relation of the level shifts to the scattering parameters

$$
\Delta E_{n L}-i \Gamma_{n L} / 2=\Omega_{n L} A_{L}
$$

TABLE II: Bohr radii and $\Omega_{n L}$ coefficients relating the level shifts to the nuclear scattering parameters for some light antiprotonic atoms.

\begin{tabular}{lcccc}
\hline \hline atom & $B[\mathrm{fm}]$ & $\Omega_{1 S}[\mathrm{keV} / \mathrm{fm}]$ & $\Omega_{2 P}\left[\mathrm{meV} / \mathrm{fm}^{3}\right]$ & $\Omega_{3 D}\left[\mu \mathrm{eV} / \mathrm{fm}^{5}\right]$ \\
\hline $\bar{p}^{1} \mathrm{H}$ & 57.63 & 0.8668 & 24.46 & 0.3591 \\
$\bar{p}^{2} \mathrm{H}$ & 43.247 & 1.539 & 77.19 & 2.012 \\
$\bar{p}^{3} \mathrm{He}$ & 19.22 & 15.59 & 3958 & 522.6 \\
$\bar{p}^{4} \mathrm{He}$ & 18.02 & 17.77 & 5125 & 770.1 \\
\hline \hline
\end{tabular}

The measurements of level shifts are equivalent to the measurements of parameters involved in the scattering amplitudes $f$, which, in the low energy expansion, are given in terms of initial $\boldsymbol{k}$ and final $\boldsymbol{k}^{\prime}$ c.m. momenta by

$$
f\left(\boldsymbol{k}, E, \boldsymbol{k}^{\prime}\right)=a_{0}(E)+3 \boldsymbol{k} \cdot \boldsymbol{k}^{\prime} a_{1}(E) .
$$

In the scattering on nuclei discussed above we use capital $A_{0}$ and $A_{1}$. In the case of $\bar{p} N$ scattering we use $a_{0}$ and $a_{1}$ which at the threshold energy become the scattering length and volume. To specify additional quantum numbers in the $\bar{p} N$ channels we use notation ${ }^{2 I+1}{ }^{2 S+1} L_{J}$, where $S, L$ and $J$ are the spin, angular and total momentum of the pair, and $I$ is its isospin. At this point we remind one that $A_{L}$ which enter relations (1) and (2) are from all short-range interactions and contain also the inner Coulomb corrections. With the procedure used here, the Coulomb field is because the target nucleus and Coulomb effects are included in the atomic-wave functions. Hence the basic scattering lengths and volumes $a_{0}$ and $a_{1}$ for the $\bar{p}-N$ systems are calculated without Coulomb corrections. The scattering lengths are defined in the baryon-baryon convention with the negative absorptive part. Thus $A_{L}=\operatorname{Re} A_{L}-i\left|\operatorname{Im} A_{L}\right|$ and a bound state close to the threshold in the $S$ wave is signaled by a large positive $\operatorname{Re} A_{0}$.

To understand elementary interactions on bound nucleons one needs to know $a_{1}(E)$ and $a_{0}(E)$ in the un-physical region of subthreshold energies. A procedure to calculate the relevant extrapolation is given in appendix A.

\section{B. Relation between level shifts and subthreshold amplitudes}

Let us consider the antiproton, bound into an atomic orbital, scattering on a nucleon, bound in a nucleus with a separation energy $E_{s}$. Atomic levels are calculated, here, in a quasi-three body system as represented in Fig. 1, and 
consisting of the antiproton, of the nucleon and of a residual nucleus, $R$. Such a model makes sense only for peripheral antiprotons and we limit ourselves to such cases. Three-body Jacobi coordinates are essential and our notation is specified in Appendix C. We intend to study energy dependence of elementary $\bar{p} N$ scattering parameters and it is important to know the energy involved in the c.m. of the interacting $\bar{p} N$ pair. It is located in the subthreshold region partly because of the bindings, nuclear $E_{s}$, atomic $E_{a}$ and partly to recoil energy, $E_{r}$, of the $\bar{p} N$ subsystem with respect to the residual nucleus. The values and ranges of the energies in question,

$$
E_{c m}=-E_{s}-E_{a}-E_{r},
$$

are indicated in Table III for the simplest first order collisions. Our discussion is limited to cases where the Born term gives a predominant result. The details of the formalism and of the calculation are given in appendices $\mathrm{A}$ and $\mathrm{C}$. Below we present the main formulas.

The inspection of Fig. 1 shows that we have to use two related pairs of Jacobi coordinate systems: $\left(\boldsymbol{k}_{12}, \boldsymbol{p}_{3}\right)$ pair useful to describe antiproton-nucleon interactions and another $\left(\boldsymbol{k}_{23}, \boldsymbol{p}_{1}\right)$ pair, useful to describe nuclear and atomic wave functions. Correspondingly, in coordinate representations, one has the $\left(\boldsymbol{r}_{12}, \boldsymbol{r}_{3}\right)$ pair and the $\left(\boldsymbol{r}_{23}, \boldsymbol{r}_{1}\right)$ one. The relation between these last two pairs is given by

$$
\boldsymbol{r}_{23}=c \boldsymbol{r}_{12}-\boldsymbol{r}_{3}, \quad \boldsymbol{r}_{1}=\gamma \boldsymbol{r}_{12}+\beta \boldsymbol{r}_{3},
$$

where

$$
c=\frac{M_{\bar{p}}}{M_{\bar{p}}+M_{N}}, \quad \gamma=\frac{M_{N}\left(M_{\bar{p}}+M_{N}+M_{R}\right)}{\left(M_{\bar{p}}+M_{N}\right)\left(M_{N}+M_{R}\right)}, \quad \beta=\frac{M_{R}}{M_{R}+M_{N}} .
$$

In this section, to simplify the notation, we use the two coordinates $\boldsymbol{r} \equiv \boldsymbol{r}_{12}$ and $\boldsymbol{\rho} \equiv \boldsymbol{r}_{23}$ that correspond to the $\bar{p}-N$ interaction range and to the $N-R$ internuclear distance, respectively (see Fig. 1).

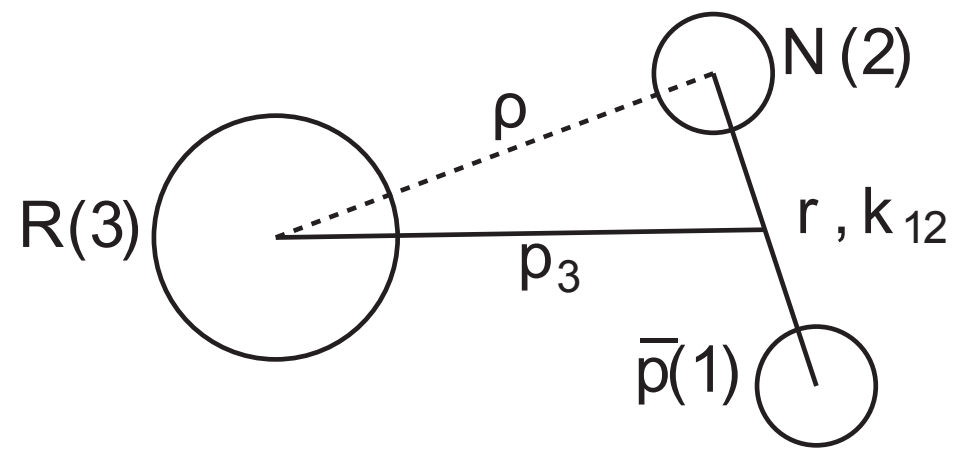

FIG. 1: Quasi-three-body system: (1) antiproton, (2) nucleon, and (3) residual system. Jacobi coordinates: momentum $\boldsymbol{p}_{3}, \boldsymbol{k}_{12}$ and space $\rho, \boldsymbol{r}$.

Let us consider the $S$-wave interactions first. The basic $\bar{p} N$ interaction potential is

$$
V_{\bar{p} N}\left(E_{c m}, S\right)=\frac{2 \pi}{\mu} \widetilde{T}_{0}\left(r, E_{c m}\right)
$$

where $\widetilde{T}_{0}\left(r, E_{c m}\right)$ is an off-shell scattering matrix given by Eq. A2. Here, isospin indices are not specified, $\mu$ is the reduced mass in the $\bar{p} N$ system. For a while, we go to local zero range limit and obtain

$$
V_{\bar{p} N}\left(E_{c m}, S\right)=\frac{2 \pi}{\mu} a_{0}\left(E_{c m}\right) \delta(\boldsymbol{r})
$$

where $a_{0}\left(E_{c m}\right)$ is the scattering amplitude at a given c.m. energy,

$$
a_{0}\left(E_{c m}\right)=\int d \boldsymbol{r} \widetilde{T}_{0}\left(r, E_{c m}\right)
$$


see Eq. (A6) for further explanations. Let us notice that potential (9), applied to $1 S$ hydrogen atom reproduces Deser-Trueman formula (1) in the leading order, up to inner electromagnetic corrections.

If the antiproton is bound into an atomic orbital, the energy shifts of upper levels (or levels of small atomic-nucleus overlap) are generated by perturbation and in the leading order

$$
\Delta E_{n L}-i \Gamma_{n L} / 2=\Sigma_{j}\left\langle\psi_{L} \varphi\left|V_{\bar{p} N_{j}}(E, S)\right| \varphi \psi_{L}\right\rangle,
$$

where the sum over $j$ extends over all nucleons of the nucleus. In general, the right-hand side of Eq. (12) consists of a complicated $4^{3}$ dimension integral which reduces to easier integrations because of the simplicity of $\vec{V}_{\bar{p} N_{i}}$. Relevant manipulations in momentum space are explained in Appendix C The main purpose of our formulation is to separate the factor of atomic-nuclear overlap and the calculation of the length $a_{0}\left(E_{c m}\right)$ averaged over the recoil energy. The wave function $\varphi$ of the struck nucleon is determined in the relative $N-R$ coordinate $\boldsymbol{\rho}$ (see Fig. 1). The last point allows us to use the proper asymptotic form of the wave function given by the separation energy. It is important as we discuss interactions localized at nuclear surfaces. The Coulomb-atomic-wave functions of given angular momentum $L$, denoted by $\psi_{L}$, are given in terms of antiproton-nucleus coordinates. The detailed development of formula (12) which, requires re-couplings of two basic Jacobi coordinate systems, is discussed in the appendix.

The recoil energy is given by $E_{r}=\boldsymbol{p}^{2} / 2 M$ where $\boldsymbol{p}$ is the total momentum of the $\bar{p}-N$ pair and $M=\mu_{R, \bar{p} N}$ is the reduced mass of this pair and of the residual nucleus $R$. As $\boldsymbol{p}$ is not a good quantum number, formula (12) involves an integral over $\boldsymbol{p}$. To obtain an intuitive picture one has to make calculations in momentum space. First let us define an average over recoil energy as

$$
\bar{a}_{0}=\int a_{0}\left(-E_{s}-E_{a}-\frac{\boldsymbol{p}^{2}}{2 M}\right) \frac{\mid \widetilde{F}_{L}\left(\left.\boldsymbol{p}\right|^{2} d \boldsymbol{p}\right.}{\int\left|\widetilde{F}_{L}(\boldsymbol{q})\right|^{2} d \boldsymbol{q}} .
$$

The extent of the recoil energies is determined by $\widetilde{F}_{L}(\boldsymbol{p})$ which is calculated in Appendix $\mathrm{C}$ and given by the formula (C4). It turns out to be a Fourier transform of the atomic and nuclear overlap

$$
F_{L}(\boldsymbol{\rho})=\varphi(\boldsymbol{\rho}) \psi_{L}(\beta \boldsymbol{\rho})
$$

with $\beta$ given in Eq. (8). It reflects the fact that the atomic wave function is given in the $R+N$ center of mass system and the nuclear wave function depends on the relative $R$ - $N$ coordinate. The level shifts are then expressed in terms of averaged scattering length and an integral over the overlap

$$
\Delta E_{n L}-i \Gamma_{n L} / 2=\frac{2 \pi}{\mu} \sum_{j}\left(\bar{a}_{0}\right)_{j} \int d \boldsymbol{\rho}\left|F_{L}(\boldsymbol{\rho})\right|^{2}
$$

TABLE III: The energy regions in $\mathrm{MeV}$ entering the $\bar{p} \mathrm{~N}$ amplitudes involved in the leading interaction terms. The first column specifies atomic states of the lightest $\bar{p}$ atoms. The numbers give average energies in the subthreshold region composed of separation and recoil components. The first number in the bracket is the proton separation energy and the second one that of the neutron. Recoil energies make no distinctions between protons and neutrons as, apart from the asymptotic region, the wave functions are not that well controlled. Differences amount to a small fraction of MeV. Numbers in parentheses indicate approximate widths of the recoil energy distributions. All numbers are rounded off to $0.1 \mathrm{MeV}$.

\begin{tabular}{llll}
\hline \hline Atom $\backslash$ State & $1 S$ & $2 P$ & $3 D$ \\
\hline $\bar{p}^{1} \mathrm{H}$ & 0 & 0 & 0 \\
$\bar{p}^{2} \mathrm{H}$ & $-2.2-8.9(5)$ & $-2.2-5.4(2)$ & $-2.2-5(1)$ \\
$\bar{p}^{3} \mathrm{He}$ & {$[-5.5-7.7] / 2-11(7)$} & {$[-5.5-7.7] / 2-8.9(2)$} & {$[-5.5-7.7] / 2-7.3(1)$} \\
$\bar{p}^{4} \mathrm{He}$ & {$[-19.8-20.6] / 2-13.8(10)$} & {$[-19.8-20.6] / 2-13.7(10)$} & {$[-19.8-20.6] / 2-13.7(10)$} \\
\hline \hline
\end{tabular}

The energies involved in Eq.13p cover some un-physical subthreshold region. The proton and neutron separation energies together with their average, the average recoil energies and the spread of recoil energies are given in Table III. These values and the separation energies indicate that three atoms ${ }^{2} \mathrm{H},{ }^{3} \mathrm{He},{ }^{4} \mathrm{He}$ cover disjoint energy regions from about -5 down to $-40 \mathrm{MeV}$. Therefore, a model is required for the subthreshold extrapolation. 
For $P$-wave-antiproton interactions we use another pseudo-potential

$$
V_{\bar{p} N}(E, P)=\frac{2 \pi}{\mu} 3 a_{1}\left(E_{c m}\right) \overleftarrow{\nabla} \delta(\boldsymbol{r}) \vec{\nabla}
$$

where $a_{1}\left(E_{c m}\right)$ is the scattering volume at a given c.m. energy. Here, spin indices are not specified. The calculations of the recoil energy and overlap integrals are performed in similar manner as for $S$ waves and details are given in the appendix C. Averaging over recoil is done as in Eq. (13), the weighting function being the same. Formulas for overlaps are more involved and may also be found in appendix $\mathrm{C}$.

\section{RESULTS OBTAINED WITH PARIS POTENTIAL MODEL}

The lowest 12 states in the two $\mathrm{H}$ and He lightest elements are listed in Table II where the appropriate overlap $\Omega_{n L}$ coefficients are presented. The standard x-ray techniques allow a direct determination of the "lower" line shapes and the extraction of the "upper" level width by the intensity loss. The experimental data from deuterium and helium consists of three level shifts and five level widths; see Tables VI VII and IX] The results from hydrogen have already been used to fix the low energy parameters of the Paris 2009 potential [6]. Here we discuss only $2 P$ shifts and $2 P, 3 D$ widths which can be described by a corrected first order perturbation.

The Paris potential is a semiphenomenological model used to describe nucleon-antinucleon scattering. It is based on fitting some 4300 data. Two versions are discussed, Paris 1999 [15] (denoted hereafter Paris 99) built to describe $\bar{p} p$ elastic and inelastic scattering data and an updated version, Paris 2009 [6] (denoted hereafter Paris 09) including also $\bar{n} p$ scattering and antiprotonic-hydrogen data. With respect to subthreshold properties these two versions differ in the position of the ${ }^{33} P_{1}$ quasibound state. Corresponding energies and widths are $\left(E_{B}, \Gamma / 2\right)=(-4.5,9) \mathrm{MeV}$ for Paris 09 and $\left(E_{B}, \Gamma / 2\right)=(-17,6.5) \mathrm{MeV}$ for Paris 99. Also, Paris 09 predicts an ${ }^{11} S_{0}$ quasibound state at energy $E=-4.8$ and width $52 \mathrm{MeV}$; there is no $S$ wave bound state in Paris 99. Another sizable difference is $a\left({ }^{3} P_{2}\right)=7.22-i 12.9 \mathrm{fm}^{3}$ for Paris 2009 and $8.44-i 8.12 \mathrm{fm}^{3}$ for Paris 99 . The preference of $a\left({ }^{3} P_{2}\right)$ cannot be definitely established [6] by the hydrogen data [9, which offers a sum of three fine structure lines.

These differences could possibly be detected in terms of the $4 P_{3 / 2}$ component in the deuteron [see below Eq. [25)]. However, Table III indicates that fine structure in the $2 P$ states in deuterium tests a narrow energy region around $-7.6 \mathrm{MeV}$ below the threshold not too far from the position of ${ }^{33} P_{1}$ quasibound state predicted by Paris 09 . If this resonance position is correct it will dominate the $4 P_{3 / 2}$ component thus reducing chances to check $a\left({ }^{3} P_{2}\right)$.

TABLE IV: $S$-wave scattering lengths in $f m$ and $P$-wave volumes in $f m^{3}$ for the Paris 99 model. The subthreshold energies, -15 and $-33 \mathrm{MeV}$ are the central energies in the $\bar{p} N$ systems in the $2 P$ atomic states for ${ }^{3} \mathrm{He}$ and ${ }^{4} \mathrm{He}$ (seeTable III).

\begin{tabular}{llll}
\hline Wave & \multicolumn{1}{c}{ At threshold } & At $-15 \mathrm{MeV}\left({ }^{3} \mathrm{He}\right)$ & At $-33 \mathrm{MeV}\left({ }^{4} \mathrm{He}\right)$ \\
${ }^{11} S_{0}$ & 0.914114 -i 0.433118 & 2.408820 -i 1.51759 & 3.90277 -i 2.78700 \\
${ }^{31} S_{0}$ & 1.116390 -i 0.368901 & 1.767780 -i 0.916545 & 1.77710 -i 1.23273 \\
${ }^{13} S_{1}$ & 1.26410 -i 0.647898 & 2.075680 -i 1.65776 & 2.71849 -i 2.52060 \\
${ }^{33} S_{1}$ & 0.454128 -i 0.355674 & 0.839587 -i 0.542044 & 1.36430 -i 0.674173 \\
\hline${ }^{13} P_{0}$ & -8.91649 -i 5.52964 & -9.49683 -i 3.63118 & -9.84196 -i 2.68574 \\
${ }^{33} P_{0}$ & 2.73731 -i $9.29717 \times 10^{-04}$ & 2.88113 -i $9.52099 \times 10^{-04}$ & 2.93514 -i $7.98058 \times 10^{-04}$ \\
${ }^{11} P_{1}$ & -3.85678 -i 0.544166 & -3.73669 -i 0.415850 & -3.76583 -i 0.376099 \\
${ }^{13} P_{1}$ & 5.11843 -i $1.81089 \times 10^{-02}$ & 5.50724 -i $2.75954 \times 10^{-02}$ & 5.63695 -i $3.26629 \times 10^{-02}$ \\
${ }^{31} P_{1}$ & 1.24447 -i 0.582186 & 1.280490 -i 0.790867 & 1.26568 -i 1.04371 \\
${ }^{33} P_{1}$ & 0.199887 -i 1.13695 & 0.361914 -i 6.58659 & -5.74199 -i 1.51859 \\
${ }^{13} P_{2}$ & -0.546355 -i 0.535542 & -0.411518 -i 0.390335 & -0.270804 -i 0.294454 \\
${ }^{33} P_{2}$ & -0.168866 -i 0.141335 & -0.129134 -i 0.146239 & -0.0741856 -i 0.162846 \\
\hline
\end{tabular}

Let us illustrate the differences between the two Paris models and the dominant factors determining level shifts in the light atoms. Table IV presents scattering parameters calculated with Paris 99 at the threshold and compared with the same parameters in the subthreshold energy region. The latter were calculated at $-15 \mathrm{MeV}$ in the case of ${ }^{3} \mathrm{He}$ and at $-33 \mathrm{MeV}$ in the case of ${ }^{4} \mathrm{He}$. These are central energies in the antiproton-nucleon systems in $2 P$ atomic states as can be seen in Table III. Analogous results for Paris 09 are given in Table V. 
TABLE V: As in Table IV but for the Paris 09 model.

\begin{tabular}{|c|c|c|c|}
\hline Wave & At threshold & ${ }^{3}$ Helium & ${ }^{4}$ Helium \\
\hline${ }^{11} S_{0}$ & 1.257770 -i 1.17353 & 0.412374 -i 5.08086 & -3.22081 -i 6.18938 \\
\hline${ }^{31} S_{0}$ & 0.751638 -i 0.560066 & 0.926035 -i 0.996672 & 1.14442 -i 1.2492 \\
\hline${ }^{13} S_{1}$ & 1.191230 -i $0.792671 \mathrm{i}$ & 1.520740 -i 1.73793 & 1.69286 -i 2.06901 \\
\hline${ }^{33} S_{1}$ & 0.608209 -i 0.435948 & 1.041720 -i 0.809038 & 1.60232 -i 1.12518 \\
\hline${ }^{13} P_{0}$ & -8.78263 -i 4.99065 & -8.84266 -i 3.40655 & -8.96530 -i 3.12356 \\
\hline${ }^{33} P_{0}$ & 2.73583 -i $0.281606 \times 10^{-02}$ & 2.87893 -i $0.317125 \times 10^{-02}$ & 2.93932 -i $0.291960 \times 10^{-02}$ \\
\hline${ }^{11} P_{1}$ & -3.58217 -i 0.320420 & -3.50523 -i 0.231635 & -3.50802 -i 0.175309 \\
\hline${ }^{13} P_{1}$ & 5.12018 -i $0.188749 \times 10^{-01}$ & 5.53295 -i $0.297656 \times 10^{-01}$ & 5.65938 -i $0.360927 \times 10^{-01}$ \\
\hline${ }^{31} P_{1}$ & 0.997969 -i 0.768087 & 0.798880 -i 0.954818 & 0.499308 -i 1.00163 \\
\hline${ }^{33} P_{1}$ & 0.285054 -i 4.107950 & -5.341040 -i 2.76101 & -4.192330 -i 0.536913 \\
\hline${ }^{13} P_{2}$ & -0.486836 -i 0.870413 & -0.494158 -i 0.768088 & -0.508217 -i 0.692099 \\
\hline${ }^{33} P_{2}$ & -0.134898 -i 0.210685 & -0.100130 -i 0.231022 & -0.0588264 -i 0.266901 \\
\hline
\end{tabular}

The atomic level shifts an widths are given by statistical averages of scattering lengths and scattering volumes. This procedure averages strong long distance effects of the pion exchange force. For example, the tremendous scattering real volume in the ${ }^{13} P_{0}$ state is off set by the real scattering volume in the ${ }^{33} P_{0}$ which has 3 times higher statistical weight. As a net effect it is the quasibound states that stick-out above the averages. On the other hand it is the spin splitting of atomic states that offers a chance to determine some specific partial waves.

The ${ }^{11} S_{0}$ displays a quasibound state in Paris 09 which is visible as strongly attractive amplitude in the deep subthreshold region met in ${ }^{4} \mathrm{He}$. This attraction is not supported by the data discussed below. On the other hand there is a strong evidence from BES [3] that such a bound state exists. This controversy requires a solution and we will suggest a way out.

Another resonant amplitude is ${ }^{33} P_{1}$ which in Paris 99 is predicted to happen in the energy region tested in ${ }^{3} \mathrm{He}$ and for Paris 09 it happens in the region of Deuteron. One consequence is that Paris 99 provides a reasonable description of deuteron shifts and Paris 09 misses the shift badly.

\section{A. Antiprotonic deuterium atoms}

The calculated and experimental shifts and widths are compared in table VI The results indicates $P$-wave dominance and strong sensitivity to the ${ }^{33} P_{1}$ quasibound state energy, see Fig. 2 for a visualization. Paris 09 solution yields an unacceptable attractive level shift and an excessive level width. That is because of strong overlap of the quasibound state with the $E_{c m}$ region characteristic for the deuterium. It is evident that bound state position of $-4.8 \mathrm{MeV}$ given by the Paris 09 model should be pushed down at least to the $[-10,-8] \mathrm{MeV}$ region which is below $-7.6 \mathrm{MeV}$ characteristic for $\bar{p} N$ c.m. energy in the $2 P$ atomic state, see Table III. This would reduce overlap of the c.m. energies with the quasibound state reducing the excessive width of the $2 P$ atomic level. In addition, locating the quasibound state below the $\bar{p} N$ energy will produce the missing repulsion to the $2 P$ level. On the other hand the ${ }^{33} P_{1}$ quasibound state generated at $-17 \mathrm{MeV}$ by Paris 99 model is too far from the $\bar{p} N$ c.m. energies and the absorption width of the $2 P$ level becomes too small. Hence, the experimental atomic levels in deuterium suggests the proper position for the ${ }^{33} P_{1}$ quasibound state to be located in between the two Paris solutions. Similar conclusion follows from Table I indicating anomalies of neutron haloes obtained in the radiochemical measurements. In the two controversial nuclei ${ }^{106} \mathrm{Cd}$ and ${ }^{112} \mathrm{Sn}$ one finds proton separation energy $S(p)=7.35$ and $7.55 \mathrm{MeV}$ and neutron separation energy $S(n)=10.87$ and $10.79 \mathrm{MeV}$, respectively. In comparison to other tested nuclei, these two, present a sizable difference in the neutron and proton separation energies. These differences are comparable to the ${ }^{33} P_{1}$ quasibound state width. Neutron haloes, tested by the radiochemical method, involve extreme nuclear surfaces and the antiproton captures occur predominantly on the valence nucleons. Characteristic $\bar{p} n$ energies in their c.m. systems are about $-16 \mathrm{MeV}$ and corresponding $\bar{p} p$ c.m. energies are about $-11 \mathrm{MeV}$. At these c.m. energies Paris 09 potential yields relative capture rates $R_{n / p} \simeq 0.9$ for both $S$ and $P$ waves in the $\bar{p} N$ systems. Actually, that value is commonly used in the analyses of the radiochemical experiments 14 but it leads to the anomalous proton haloes in ${ }^{106} \mathrm{Cd}$ and ${ }^{112} \mathrm{Sn}$ nuclei. These anomalous results may be understood if the resonant position is close to the $\bar{p} p$ c.m. energies and far from the $\bar{p} n$ c.m. energies. Such situation may favor $\bar{p} p$ captures imitating proton nuclear haloes. To meet this, the ${ }^{33} P_{1}$ state 
should to be located in $[-11,-9] \mathrm{MeV}$ segment.

TABLE VI: Results for $2 P$-deuterium level corrections in meV calculated with the spin averaged amplitudes of the Paris 09 potential. The second and third column numbers are the real and twice the imaginary part of the average complex shift $\Delta(27)$. Numbers in curly brackets are results with the Paris 99 potential.

\begin{tabular}{lcc}
\hline \hline order & Shift & Width \\
\hline$S$ wave & $100\{113\}$ & $210\{145\}$ \\
$P$ wave & $-9\{58\}$ & $365\{206\}$ \\
\hline Sum & $91\{171\}$ & $575\{341\}$ \\
Data [8, 9] & $243 \pm 26$ & $489 \pm 30$ \\
\hline \hline
\end{tabular}

\section{B. Helium atoms}

Experiments measure lower $2 P$ and and upper $3 D$ atomic levels. Bulk of the interaction happens in a low density region, although Helium nuclei have no real surface region. Multiple scattering is negligible in the $3 D$ states but sizable in the $2 P$ states. What matters most is a proper single nucleon wave function. Here, we use Eckart functions [21, 22] which have asymptotic given by separation energies $E_{s}$ and a phenomenological form at short distances

$$
\varphi(r)=[1-\exp (-\widetilde{\beta} r)]^{4} \frac{\exp (-\widetilde{\alpha} r)}{r}
$$

where $\widetilde{\alpha}=\sqrt{2 \mu_{R, N} E_{s}}$. The short distance parameter $\widetilde{\beta}$ is extracted from electron or pion scattering experiments and fixed to reproduce the zeros of charge form-factors. Thus for ${ }^{3} \mathrm{He}, \widetilde{\alpha}=0.45, \widetilde{\beta}=1.753$ [23, 24], and for ${ }^{4} \mathrm{He}, \widetilde{\alpha}=0.846, \widetilde{\beta}=1.20\left[22\right.$, all in $\mathrm{fm}^{-1}$ units.

TABLE VII: Leading order calculations in $\mathrm{eV}$ for $2 P$ and in meV for $3 D$ (widths only) level corrections in ${ }^{3} \mathrm{He}$ obtained with the spin averaged amplitudes of the Paris 09 potential. Numbers in curly brackets are obtained with the Paris99 potential.

\begin{tabular}{lccc}
\hline & 2P shift & 2P width & 3D width \\
\hline$S$ wave & $6.68\{9.22\}$ & $17.5\{11.5\}$ & $0.69\{0.49\}$ \\
$P$ wave & $-6.36\{1.44\}$ & $15.0\{26.9\}$ & $1.46\{2.08\}$ \\
Sum & $0.31\{10.71\}$ & $32.5\{38.4\}$ & $2.15\{2.57\}$ \\
Data 11 & $17 \pm 4$ & $25 \pm 9$ & $2.14 \pm 0.18$ \\
\hline \hline
\end{tabular}

Reference 25] finds (with a different $N \bar{N}$ potential) a $2 \%$ higher order corrections to the $2 P$ levels in deuterium. Such corrections are of the same magnitude with the Paris potentials and would not change our conclusions. On the other hand in Helium we find sizable multiple scattering corrections. A method to sum the multiple scattering expansion series is presented in appendix $\mathrm{D}$. Here we present results.

TABLE VIII: As in Table VII but only for $2 P$ level including higher order corrections. Now the contribution of the $P$ wave interaction depends also on $S$ wave interaction as a result of multiple scattering summation method.

\begin{tabular}{lcc}
\hline & 2P shift & 2P width \\
\hline$S$ wave & $6.66\{7.83\}$ & $12.3\{7.70\}$ \\
$P$ wave & $-5.20\{4.75\}$ & $19.1\{22.13\}$ \\
Sum & $1.46\{12.59\}$ & $31.4\{29.8\}$ \\
Data [1] & $17 \pm 4$ & $25 \pm 9$ \\
\hline \hline
\end{tabular}


Tables VII and VIII compare first order and higher order results. The differences are fairly small and also difficult to interpret in simple terms. In general the widths are suppressed which is a standard effect of strong absorption. Small changes in level shifts reflect interference of first order scattering parameters $a$ and second order $a^{2}$ which depends on the signs of real parts. The average scattering lengths yield repulsive shifts $\left(R e a_{0}>0\right)$ but scattering volumes generate attraction $\left(R e a_{1}<0\right)$ below the quasibound state and repulsion $\left(R e a_{1}>0\right)$ above this state. Thus the interference pattern of the single scattering and double scattering terms depends on the $\bar{p} N$ c.m. energy.

Paris 99 model is fairly consistent with the Helium data while Paris 09 misses the repulsion in the $2 P$ atomic state. The downward shift of ${ }^{33} P_{1}$ required by the deuteron levels does not remove the inconsistency. Apparently there is another source of the difficulty and the ${ }^{4} \mathrm{He}$ atoms indicate a new possibility.

TABLE IX: As in Table VII but for the ${ }^{4} \mathrm{He}$ atoms.

\begin{tabular}{lccc}
\hline & 2P shift & 2P width & 3D width \\
\hline$S$ wave & $9.72\{17.6\}$ & $26.0\{19.8\}$ & $0.66\{0.50\}$ \\
$P$ wave & $-9.01\{-10.4\}$ & $14.9\{14.8\}$ & $0.91\{0.91\}$ \\
Sum & $0.708\{7.2\}$ & $40.9\{34.6\}$ & $1.57\{1.41\}$ \\
Data 11] & $18 \pm 2$ & $45 \pm 5$ & $2.36 \pm 0.10$ \\
\hline \hline
\end{tabular}

TABLE X: As in Table VIII but for the ${ }^{4} \mathrm{He}$ atoms.

\begin{tabular}{lcc}
\hline & 2P shift & 2P width \\
\hline$S$ wave & $8.94\{12.3\}$ & $14.7\{10.4\}$ \\
$P$ wave & $-8.71\{-10.9\}$ & $19.0\{18.6\}$ \\
Sum & $0.23\{1.4\}$ & $33.7\{29.0\}$ \\
Data 11] & $18 \pm 2$ & $45 \pm 5$ \\
\hline \hline
\end{tabular}

Tables $[\mathrm{IX}$ and $\mathrm{X}$ compare first order and higher order results, respectively. Both fail to reproduce the repulsive level shifts. Difficulties of the Paris potential rise with the increasing distance from the nucleon-antinucleon threshold. The ${ }^{4} \mathrm{He}$ atom presents an extreme case which is not matched even in heavy antiprotonic atoms. Because of the peripheral nature of nuclear antiproton captures the nucleon separation energies as high as $21 \mathrm{MeV}$ are met very rarely. In some sense the ${ }^{4} \mathrm{He}$ atom is located on the boundary of unknown $N \bar{N}$ subthreshold c.m energies. Many models of $N \bar{N}$ have predicted deeply quasibound states which historically have been viewed with scepticism as nothing was determined in direct experiments. Furthermore the meson exchange interactions are not very reliable at short distances. The Paris 09 potential also generates such a deep and broad state at about $-80 \mathrm{MeV}$ in the isospin 1 state. Visualization of the related amplitudes may be found in reference [26] which shows that this state dominates the $S$-wave subthreshold scattering in this deep region. Now, the difficulty in the understanding of He atomic data would be solved immediately if the position of this quasibound state is shifted up by some $20 \mathrm{MeV}$. Both, the missing repulsion and somewhat weak absorption strength will reach consistency with the data.

\section{The $R_{n / p}$ ratios}

Different but related experiments had been performed in the early LEAR era. These studied captures of stopped antiprotons in deuterium and helium chambers and measured relative rates of antiproton captures by neutrons and by protons. Such ratios are essential for studies of the neutron haloes in heavier nuclei. Table XI gives $R_{n / p}$, the ratio of basic $\bar{p} n$ capture rate to $\bar{p} p$ capture rate. It indicates consistency of Paris 09 potential with the $R_{n / p}$ results in ${ }^{3} \mathrm{He}$ and ${ }^{4} \mathrm{He}$. The $R_{n / p}$ was calculated in $2 P$ atomic state that is likely to be the state of capture for $70 \%$ of all antiprotons that reach low level atomic states [27. These findings are also correct if a sizable fraction of antiproton absorption occurs from higher atomic $n P$ states.

On the other hand, the deuterium calculations given in table XI have no direct relation to the data as the capture is likely to happen from many $n S$ atomic states. The $n S$ results put into brackets are given by the Paris potentials, but calculated under the assumption that captures in $n S$ deuterium levels happen predominantly in the $\bar{p} N S$ waves. This assumption is based on calculations of Ref. [25. Here, we are unable to offer the atomic cascade details and 
predict the fraction of captures from $n P$ states relative to captures from the $n S$ atomic states. Nevertheless, Table XI allows the conclusion that Paris 09 sets proper limits on the $R_{n / p}$ in deuterium too. Results with Paris 99 are also satisfactory although too high for the $\bar{p}^{3} \mathrm{He}$ and $\bar{p}{ }^{4} \mathrm{He}$ atoms.

TABLE XI: The $R_{n / p}$, ratios. Second column gives experimental results from antiprotons stopped in bubble chambers. Third and fourth columns give the ratios calculated with the two versions of the Paris potential. It is assumed that capture occurs from $n P$ atomic levels. The experimental ratio in ${ }^{3} \mathrm{He}[30$ ] is given per target $0.35(7)$. Here it is normalized to values per single nucleon, hence a factor 2. Results for captures in deuterons from $n S$ states are given into angle brackets.

\begin{tabular}{llccc}
\hline \hline Atom & Experiment & Paris 09 & Paris 99 \\
\hline $\bar{p}^{2} \mathrm{H}$ & {$[28$} & $0.81(3)$ & $1.09\langle 0.55\rangle$ & $0.84\langle 0.61\rangle$ \\
$\bar{p}^{2} \mathrm{H}$ & 29 & $0.749(18)$ & $1.09\langle 0.55\rangle$ & $0.84\langle 0.61\rangle$ \\
$\bar{p}^{3} \mathrm{He}$ & {$[30$} & $0.70(14)$ & .65 & 1.00 \\
$\bar{p}{ }^{4} \mathrm{He}$ & 30 & $0.48(3)$ & .48 & 0.59 \\
\hline \hline
\end{tabular}

\section{Hyperfine structure}

All $N \bar{N}$ potentials are strongly spin dependent. That generates hyperfine structure of atomic levels built by strong interactions and superposed on the electromagnetic structure. Below we present the isospin and spin structure of the basic amplitudes involved in the iso-spin and spin states.

The isospin structure is given by

$$
A(\bar{p} p)=[a(I=0)+a(I=1)] / 2 ; \quad A(\bar{p} n)=a(I=1) .
$$

For the $1 S$ atomic level, deuteron spin 1 adds to antiproton spin $1 / 2$ to total spin doublet and total spin quartet states. For these, the appropriate amplitudes for $S$-wave antiproton-deuteron interaction are denoted $A\left(\lambda S_{\widetilde{J}}\right)$ where $\lambda=2,4$ denotes doublet or quartet and $\widetilde{J}$ denotes total angular momentum of the three particles. The notation for the nucleon-antinucleon pair was defined in Sec. II A viz. ${ }^{2 I+1} 2 S+1 L_{J}$. The antiproton interacts on the neutron and proton of the deuteron and we just add both interactions and in the notation used below, in Eqs. 20, -26 , we suppress the upper isospin subscript and, following Eq. (18), we define,

$$
a\left({ }^{2 S+1} L_{J}\right)=\frac{1}{2} a\left({ }^{12 S+1} L_{J}\right)+\frac{3}{2} a\left({ }^{32 S+1} L_{J}\right)
$$

One has

$$
A\left(2 S_{1 / 2}\right)=\frac{3}{4} a\left({ }^{1} S_{0}\right)+\frac{1}{4} a\left({ }^{3} S_{1}\right) ; \quad A\left(4 S_{3 / 2}\right)=a\left({ }^{3} S_{1}\right) .
$$

For $P$-wave interactions in the $1 S$ state one has expressions similar to those of Eq. (20),

$$
A\left(2 P_{1 / 2}\right)=\frac{3}{4} a\left({ }^{1} P_{1}\right)+\frac{1}{4} a\left({ }^{3} P_{1}\right) ; \quad A\left(4 P_{3 / 2}\right)=a\left({ }^{3} P_{1}\right) .
$$

In $2 P$ atomic levels, the amplitudes given by Eq. 18 and $(19)$ are mixed in the total $\widetilde{J}=1 / 2,3 / 2,5 / 2$ three particle states. However, in both Paris potentials, the splitting from the $S$-wave interactions is minute and the main effect comes from the $P$-wave antiproton-nucleon interactions. For the two doublet and three quartet states in the $2 P$ atomic levels, one requires additional terms given by $P$-wave $N \bar{N}$ amplitudes. In doublet the combinations are

$$
\begin{gathered}
A\left(2 P_{1 / 2}\right)=\left[9 a\left({ }^{1} P_{1}\right)+a\left({ }^{3} P_{0}\right)+2 a\left({ }^{3} P_{1}\right)\right] / 12, \\
A\left(2 P_{3 / 2}\right)=\left[9 a\left({ }^{1} P_{1}\right)+\frac{1}{2} a\left({ }^{3} P_{1}\right)+\frac{5}{2} a\left({ }^{3} P_{2}\right)\right] / 12,
\end{gathered}
$$


and for the spin quartet

$$
\begin{gathered}
A\left(4 P_{1 / 2}\right)=\frac{2}{3} a\left({ }^{3} P_{0}\right)+\frac{1}{3} a\left({ }^{3} P_{1}\right), \\
A\left(4 P_{3 / 2}\right)=\frac{5}{6} a\left({ }^{3} P_{1}\right)+\frac{1}{6} a\left({ }^{3} P_{2}\right), \\
A\left(4 P_{5 / 2}\right)=a\left({ }^{3} P_{2}\right) .
\end{gathered}
$$

The hyperfine structure level shifts and widths fullfil the relation

$$
\Delta=\frac{\sum_{k=1}^{5}\left(2 \widetilde{J}_{k}+1\right) \Delta_{k}}{\sum_{k=1}^{5}\left(2 \widetilde{J}_{k}+1\right)},
$$

where the complex shifts $\Delta_{k}$ are given in Table XII. The summation extends over all five states specified in Eqs. (22) - (26) and involves both $S$ and $P$ wave interactions. We find that the average $\Delta$ differs by less than $8 \%$ from the shift obtained by the spin averaged $\bar{p} N$ scattering parameters displayed in Table VI. The numbers presented in Table XII do not contain electromagnetic splittings which are also of the order of $100 \mathrm{eV}[8]$.

TABLE XII: Hyperfine structure splittings, $\Delta_{k}=\epsilon_{k}-i \Gamma_{k} / 2$ in $\mathrm{eV}$, predicted for $2 P$ states in deuterium.

\begin{tabular}{cccccc}
\hline \hline & \multicolumn{3}{c}{ Paris 09 } & \multicolumn{3}{c}{ Paris 99 } \\
\hline$k$ & state & $\epsilon_{k}$ & $\Gamma_{k}$ & $\epsilon_{k}$ & $\Gamma_{k}$ \\
\hline 1 & $2 P_{1 / 2}$ & -21 & 670 & 204 & 459 \\
2 & $2 P_{3 / 2}$ & 33 & 203 & 101 & 323 \\
\hline 3 & $4 P_{5 / 2}$ & 81 & 282 & 89 & 162 \\
4 & $4 P_{3 / 2}$ & 241 & 1250 & 337 & 630 \\
5 & $4 P_{1 / 2}$ & 54 & 620 & 79 & 344 \\
\hline \hline
\end{tabular}

The experiment gives essentially no hyperfine structure splitting but offers a good check on the widths controlled by direct line structure and the x-ray intensity loss. Both Paris potentials generate splitting which is narrow in relation to the level widths but built at an incorrect average level shift (see Table VI).

One level, the $4 P_{3 / 2}$ is singled out and located separately. Being very broad it can also be missed in the spectrum. It reflects strong overlap of $\bar{p} N$ c.m. energy with the quasibound state spectral density. As discussed already, a downward, few $\mathrm{MeV}$ shift of the quasibound state, would still enlarge the width and reduce attraction removing practically the fine structure splitting. It must be added that this quasibound state shift will also massively repulse the average shift given in Table VI as required by the data. The scenario discussed here is plotted in Fig. 2.

On the other hand Paris 99 gives a group of five lines that seem close to data both in terms of cumulated widths and average shift. In conclusion we see that the data are not consistent with the $-4.5 \mathrm{MeV}{ }^{33} P_{1}$ quasibound state but may tolerate this state if its binding is larger.

\section{CONCLUSIONS}

Antiprotonic atomic levels characterized with very small nuclear-atom overlap are a powerful method to study antiproton-nucleon amplitudes below the antiproton-nucleon threshold. In this way one is able to study the structure of the amplitudes in the region reaching down to some $-40 \mathrm{MeV}$ below the threshold.

Comparison of the level shifts and widths in deuterium and helium atoms with the predictions of Paris potential model yields following conclusions.

(1) The Paris 2009 potential offers $S$-wave antiproton-nucleon amplitudes dominated, just below the threshold, by a broad quasibound state in ${ }^{11} S_{0}$ wave. Interactions in this wave generate strongly repulsive atomic levels in the light atoms. On the other hand, the $P$-wave interactions are attractive, on average, unless there exist very deeply bound $P$-wave quasibound states which does not happen in both models. The balance between $S$-wave repulsion and 


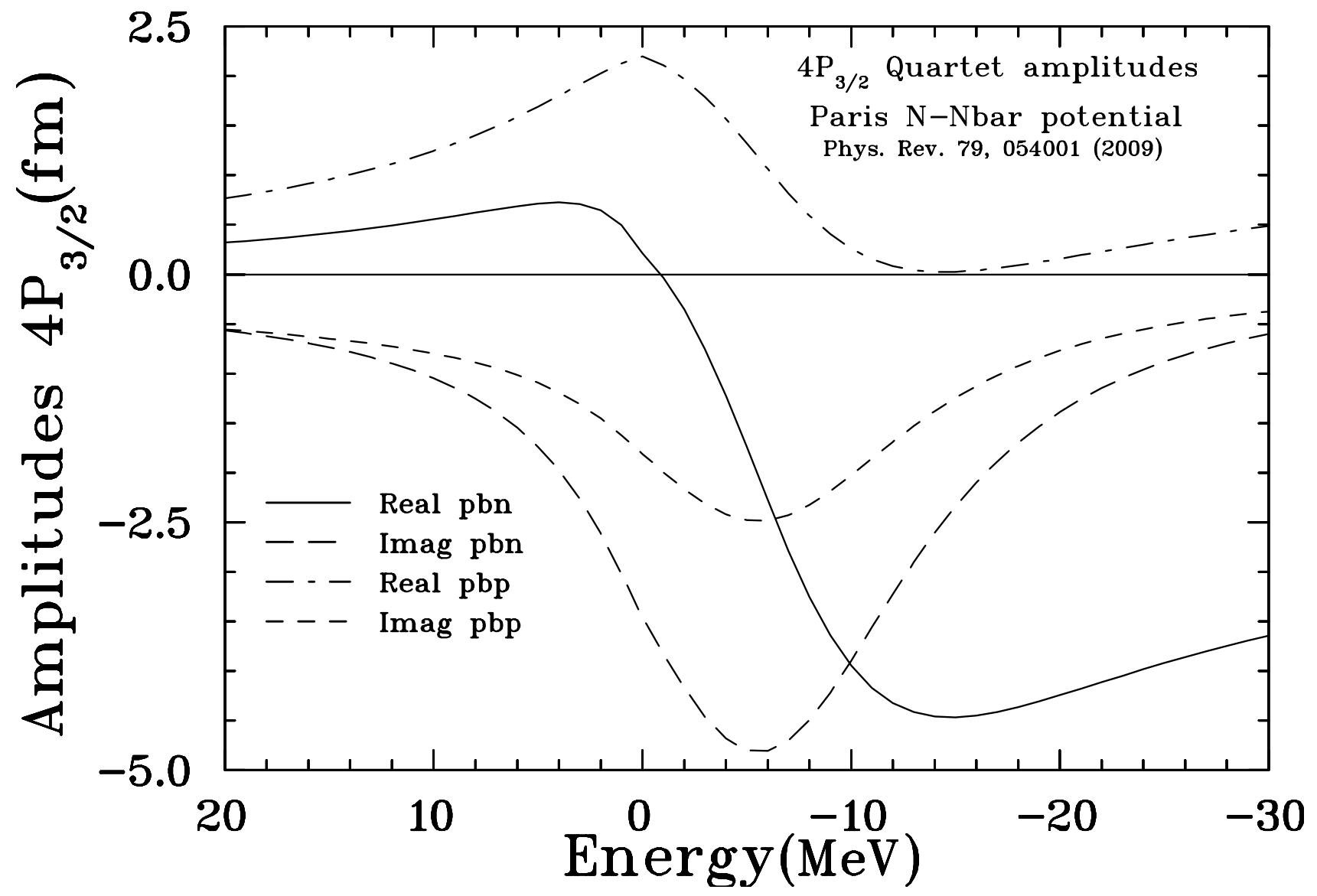

FIG. 2: Subthreshold amplitudes generating the $4 P_{3 / 2}$ hyperfine structure component in deuterium. With Paris 09 solution this amplitude is strongly dominated by the resonant $a\left({ }^{33} P_{1}\right)$ amplitude. Relevant $\bar{p} N$ c.m. energies fall in the region $-7.6 \pm 1 \mathrm{MeV}$ and the resonance position is $-4.8 \mathrm{MeV}$. Downward shift of the resonance position by a $-4 \mathrm{MeV}$ or more will strongly reduce the attraction calculated in this component. In this way the hyperfine structure splitting is practically nullified.

$P$-wave attraction gives an indication of the uncertain position of the ${ }^{33} P_{1}$-quasibound state predicted by two Paris potential models. Alas, the repulsion from the ${ }^{11} S_{0}$ wave is not strong enough to explain the experimental, strongly repulsive, levels. The strongest discrepancy happens in the ${ }^{4} \mathrm{He}$ atom where the energies in the $\bar{p} N$ subsystems reach the lower limit of about $-40 \mathrm{MeV}$. The data require a strong repulsion which is not given by the model and some enhancement of the absorption strengths predicted by the model. All this demands a new phenomenon in the region below $-40 \mathrm{MeV}$. It, in fact, exists in the Paris 09 model and is given by the isospin 1 quasibound state predicted at $-80 \mathrm{MeV}$. Consistency with the data requires a shift of this state up to about $-60 \mathrm{MeV}$.

(2) The level shifts in light atoms require some enhancement of nuclear repulsion. This observation is contrary to the one found in medium and heavy atoms where attractive antiproton optical potentials are required. That happens with phenomenological potentials [31] and optical potentials based on Paris $\bar{p} N$ amplitudes [32. The scenario in light and heavy nuclei differ as lower levels in heavier atoms involve higher nuclear densities and the resulting level shifts are far from the Born approximations. Nuclear many body effects matter. It is well understood that the exclusion principle operating in nuclear matter may dissolve or push up the $\bar{p} N$ quasibound state. This may induce the missing attraction. Reference 32 includes this effect on a Fermi gas model with Fermi momentum $K_{f} \sim \rho^{1 / 3}$ but finds it not strong enough to bring agreement with the data. A more extreme approach was studied in Refs. 33, 34, where a different form of $K_{f}$ was used and a kind of effective self-consistent antiproton nucleon scattering amplitude $T_{\text {eff }}$ was calculated. That may generate required attraction but the price to pay is a sizable uncertainty in the method used to calculate $T_{\text {eff }}$ at the nuclear surfaces. It is clear that the problem is not straightforward. In this work we try to check the basic $\bar{p} N$ subthreshold amplitudes in a simple situation. Studies of heavier atoms and the physics of the involved nuclear surface structure would come next.

(3) The $P$-wave antiproton-nucleon interaction of Paris potential generates a quasibound state in ${ }^{33} P_{1}$ state. Its position is unstable and occurs at $-17 \mathrm{MeV}$ in the potential version Paris 99 and at $-4.5 \mathrm{MeV}$ in that of Paris 09 . Neither solution reproduces all atomic data. Consistency with the ${ }^{2} \mathrm{H},{ }^{3} \mathrm{He}$ atomic levels, and understanding of the 
$R_{n / p}$ anomalies seen in radiochemical experiments require the state to be located in the [-11, -9$] \mathrm{MeV}$ region.

(4) Older data related to bubble chamber measurements of relative $n \bar{p}$ and $p \bar{p}$ capture rates $R_{n / p}$ are consistent with Paris 09 in all elements. This reflects a fair success of the model in description of the average subthreshold annihilation rates.

(5) The ${ }^{33} P_{1}$ quasibound state dominates the fine structure $4 P_{3 / 2}$ component of $2 P$ atomic state in the antiprotonic deuterium. Unfortunately it is likely to be very broad. Measurement of the $4 P_{3 / 2}$ fine structure would be valuable and would fix the energy of ${ }^{33} P_{1}$ quasibound state.

(6) Let us compare the models : on the basis of hydrogen and helium atomic data it seems that Paris 99 model has some advantage over the later Paris 09 version. Definitely the level shifts are better because of the prediction of the strongly bound ${ }^{33} P_{1} \bar{N} N$ state. The same state, bound weakly by the Paris 09 potential, presents difficulties and if this state really exists it should be bound more strongly. On the other hand Paris 09 offers some advantages. It was based on the addition of anti-neutron scattering data and the experimental neutron/proton capture rates $R_{n / p}$ are better reproduced. That offers an advantage for the PUMA project. Another advantage of the latter potential is a better description of the BES Collaboration enhancement results [7].

Both models have problems with the ${ }^{11} S_{0}$ state which begin already at the level of antiprotonic hydrogen. Calculated level shifts are almost twice as large as the experimental ones [6]. The description of $\bar{N} N$ interaction in this state is particularly difficult as the well known pion exchange potential is extremely strong and may generate a number of quasibound states. Various ways to moderate this potential at very short distances lead to instabilities of the bound state energies. Now, Paris09 potential offers a broad but very weakly bound state and a deeply bound one. Probably both states have to be readjusted.

To summarize: there is an indication that Paris 09 may be a starting point to offer successful description of atomic, bubble chamber, and radiochemical data provided the position of $P$-wave baryonium is shifted down by about a few $\mathrm{MeV}$ and the deeply bound $S$ state is pushed up by some $20 \mathrm{MeV}$. That requires an update of this potential model and the work is in progress.

\section{Acknowledgements}

We wish to thank Jaume Carbonell for helpful discussions and Detlev Gotta for comments and advice on experimental side. This study was initiated by Alexandre Obertelli and PUMA project at CERN. The collaboration was supported by COPIN-IN2P3 agreement No. 05-115.

\section{Appendix A: Half-off-shell $N \bar{N}$ interactions}

For atomic calculations one needs the off-shell extension of the scattering amplitude in the energy as well as in the momentum variables. The most general extension for $S$ waves is given by

$$
T\left(k, E, k^{\prime}\right)=\frac{\mu_{N \bar{N}}}{2 \pi} \int \psi_{o}(r, k) V_{N \bar{N}}(r, E) \Psi\left(r, E, k^{\prime}\right) r^{2} d r,
$$

where $\Psi\left(r, E, k^{\prime}\right)$ is the full outgoing wave calculated with the regular free wave $\psi_{o}(r, k)=\sin (r k) /(r k)$. The normalization factor is chosen to produce $T\left(k, E, k^{\prime}\right)$ equal to the scattering lengths. In Eq. (A1) the momentum $k^{\prime}$ is not related to the energy $E$. The Fourier-Bessel double transform of $T\left(k, E, k^{\prime}\right)$ would generate a nonlocal $\widetilde{T}\left(r, E, r^{\prime}\right)$ matrix in the coordinate representation. Such involved calculations do not seem necessary as the experimental data are not that precise. We resort to a simpler procedure, standard in nuclear physics, and for an application in the antiproton physics see Ref. 33. The subthreshold scattering amplitudes are calculated in terms of an effective $\widetilde{T}(r, E)$ matrix defined in the coordinate representation by

$$
\widetilde{T}(r, E)=\frac{\mu_{N \bar{N}}}{2 \pi} V_{N \bar{N}}(r, E) \frac{\Psi\left(r, E, k^{\prime}(E)\right)}{\psi_{o}\left(r, k^{\prime}(E)\right)},
$$

with $k^{\prime}(E)=\sqrt{2 \mu_{N \bar{N}} E}$. In this equation $\Psi\left(r, E, k^{\prime}(E)\right)$ is the solution of the Lippman-Schwinger equation $\Psi=\psi_{o}+G^{+} V \Psi$ or of an equivalent Schrödinger equation. To avoid confusion in the notation we specify the $\Psi$ in the simplest $S$ wave state. It is the radial scattering solution regular at the origin which behaves asymptotically as 


$$
\Psi\left(r, E, k^{\prime}(E)\right) \sim \frac{i}{2 k^{\prime} r}\left[e^{-i k^{\prime} r}-S e^{i k^{\prime} r}\right]
$$

where $S=\exp (i 2 \delta)$ is the scattering matrix. The $\widetilde{T}(r, E)$ is a local equivalent of the nonlocal T matrix in the sense that its matrix elements fulfill the relation (obtained from Eqs. (A1) and A2 with $k^{\prime}$ replaced by $k^{\prime}(E)$ ) $T\left(k, E, k^{\prime}(E)\right)=\int d r r^{2} \psi_{o}(r, k) \widetilde{T}(r, E) \psi_{o}\left(r, k^{\prime}(E)\right)$ valid in a narrow subthreshold region where the last integral is convergent. For positive energies this equation is not practical because of zeros in the denominator of Eq. A2 which occur at multiplicities of $k^{\prime}=\pi / r$. One could nevertheless use it for $k^{\prime}<\pi / r_{\text {max }}$ where $r_{\text {max }}$ is the distance at which the potential is cut-off. In this narrow region one finds

$$
T\left(k, E, k^{\prime}\right)=\int \psi_{o}(r, k) \widetilde{T}(r, E) \psi_{o}\left(r, E, k^{\prime}\right) r^{2} d r
$$

thus, in this case, the $\widetilde{T}(r, E)$ reproduces the half- and full-off-shell amplitudes, allowing to obtain a numerical continuity in calculations. One needs also the Fourier transform of $\widetilde{T}(r, E)$,

$$
T(\kappa, E)=\int d \boldsymbol{r} \widetilde{T}(r, E) \frac{\sin (\kappa r)}{\kappa r},
$$

and the relevant $S$-wave scattering amplitudes is given by

$$
a(E)=T(0, E)=\int d \boldsymbol{r} \widetilde{T}(r, E) .
$$

The main step is to use equation A2 for negative energies with $V_{N \bar{N}}(r, E)$ given by the Paris model. It is used in the Schrödinger equation to calculate $\Psi\left(r, E, k^{\prime}(E)\right)$. Formula A2 requires numerical care and cutting the potential tail at very large distances is recommended. This point, related to the question of relatively long ranged pion-exchange forces particularly strong in the nucleon-antinucleon systems, is discussed below.

\section{Appendix B: The $N \bar{N}$ force range effects}

With no knowledge of the full off-shell $T\left(r^{\prime}, E, r\right)$ matrix, one cannot utilize the power of Faddeev 3-body equations. On the level of the pseudopotential method, used here, the question is: what is a better description, either the local zero-range potential of Eq. 10) or a type of "folded" potential given by Eq. (9), with the range given by the half-off shell $\widetilde{T}(E, r)$. We tried both with the result that the folded potential with the range involved within $\widetilde{T}(E, r)$ is not acceptable. In particular the range in the absorptive part in $\widetilde{T}(E, r)$ is fairly long from joint impact of short range effects and long range pion exchange. The mean square radius of this range defined by

$$
R_{m s}=\sqrt{\frac{\operatorname{Im}\left[\int d \boldsymbol{r} \widetilde{T}(r, E) r^{2}\right]}{\operatorname{Im}\left[\int d \boldsymbol{r} \widetilde{T}(r, E)\right]}}
$$

amounts to about $1.3 \mathrm{fm}$, in both models, while the range of the basic nucleon-antinucleon potential is about 0.9 fm. In helium atoms, for $2 P$ states, the range effects are moderate and both Eqs. (10) and (9) yield comparable results. However, the range involved in $\widetilde{T}(E, r)$ makes tremendous difference in the upper $3 D$ level widths given predominantly by $R_{m s}^{4}$. In those levels, the widths depend on the well known asymptotic of nuclear wave functions and are given essentially by the first order scattering terms. The experimental data rules out folded potential based on the $\widetilde{T}(r, E)$ by several standard deviations.

A similar result was found in Refs. [33, 34] where a simple two term separable potential for the $N-\bar{N}$ interactions is studied with some form-factors $v(r)$ representing effects of pion exchange. The full off-shell $T$ matrix involves terms $T\left(r^{\prime}, E, r\right) \sim v\left(r^{\prime}\right) t(E) v(r)$ where the $t(E)$ matrix is based on the Dover-Richard $N \bar{N}$ potential. Such terms in the few-nucleon or nuclear systems yield effective interaction range, determined by $v(r)^{2}$, roughly one half of the range given by the half off-shell $\widetilde{T}(r, E)$. 
We conclude that localized potential $(10)$, with range effect hidden in $a\left(E_{c m}\right)$, is more realistic in the overall approach and it was used in the calculations. However, with future $N-\bar{N}$ potentials, of properly fixed quasibound states positions, it is advisable to use fully off-shell input which will adapt itself better to states of different atomic angular momenta.

\section{Appendix C: Averaging over recoil energy}

Following Sec. IIB we consider the 3-body system depicted in Fig. 1 and denote the antiproton, the struck nucleon and the residual nucleus as particles 1, 2 and 3, respectively. We adopt the Jacobi coordinates $\boldsymbol{k}_{i j}, \boldsymbol{p}_{m}(i, j, m=1,2,3$, $i<j$ ) and in coordinate representation we use $\boldsymbol{r}_{i j}, \boldsymbol{r}_{m}$, but to simplify the notation, we specify only the two coordinates $\boldsymbol{r}$ and $\boldsymbol{\rho}$ shown in the figure.

For $S$-wave zero-range interactions the relevant antiproton-nucleon operator in momentum space is

$$
\widehat{a}_{0}=a_{0}\left(E_{B}-\frac{\boldsymbol{p}_{3}^{2}}{2 \mu_{12,3}}\right) \delta\left(\boldsymbol{p}_{3}-\boldsymbol{p}_{3}^{\prime}\right),
$$

where $\mu_{12,3}$ is the reduced mass of the 12 pair and particle 3 . With the wave functions $\widetilde{\varphi}\left(\boldsymbol{k}_{23}\right)$ and $\widetilde{\psi}\left(\boldsymbol{p}_{1}\right)$ one has the first order expectation value

$$
\left\langle\widehat{a}_{0}\right\rangle=\int d \boldsymbol{k}_{12} d \boldsymbol{p}_{3} d \boldsymbol{k}_{12}^{\prime} d \boldsymbol{p}_{3}^{\prime} \widetilde{\varphi}\left(\boldsymbol{k}_{23}\right) \widetilde{\psi}\left(\boldsymbol{p}_{1}\right) a_{0}\left(E_{B}-\frac{\boldsymbol{p}_{3}^{2}}{2 \mu_{12,3}}\right) \delta\left(\boldsymbol{p}_{3}-\boldsymbol{p}_{3}^{\prime}\right) \widetilde{\varphi}^{*}\left(\boldsymbol{k}_{23}^{\prime}\right) \widetilde{\psi}^{*}\left(\boldsymbol{p}_{1}^{\prime}\right) .
$$

To exploit the $\delta\left(\boldsymbol{p}_{3}-\boldsymbol{p}_{3}^{\prime}\right)$ function, we use another pair of Jacobi coordinates

$$
\boldsymbol{p}_{1}=-\boldsymbol{k}_{12}-c \boldsymbol{p}_{3} ; \quad \boldsymbol{k}_{23}=-\beta \boldsymbol{k}_{12}+b \boldsymbol{p}_{3},
$$

where $b=1-c \beta$.

Now we define a function $\widetilde{F}$

$$
\widetilde{F}\left(\boldsymbol{p}_{3}\right)=\int d \boldsymbol{k}_{12} \widetilde{\varphi}\left(-\beta \boldsymbol{k}_{12}+b \boldsymbol{p}_{3}\right) \widetilde{\psi}\left(-\boldsymbol{k}_{12}-c \boldsymbol{p}_{3}\right),
$$

which allows to present expectation value $\left\langle\widehat{a}_{0}\right\rangle$ as

$$
\left\langle\widehat{a}_{0}\right\rangle=\bar{a}_{0} \int d \boldsymbol{p}_{3}\left|\widetilde{F}\left(\boldsymbol{p}_{3}\right)\right|^{2}
$$

where

$$
\bar{a}_{0}=\int d \boldsymbol{p}_{3} a_{0}\left(E_{B}-\frac{\boldsymbol{p}_{3}^{2}}{2 \mu_{12,3}}\right)\left|\widetilde{F}\left(\boldsymbol{p}_{3}\right)\right|^{2} / \int d \boldsymbol{p}_{3}\left|\widetilde{F}\left(\boldsymbol{p}_{3}\right)\right|^{2} .
$$

This corresponds to subthreshold value of the scattering length averaged over some region of recoil energies. The last expression becomes more intuitive in the coordinate representation. One obtains Fourier transform of $\widetilde{F}\left(\boldsymbol{p}_{3}\right)$ expressed by

$$
F(\boldsymbol{\rho})=\varphi(\boldsymbol{\rho}) \psi(a \boldsymbol{\rho})
$$

i.e. it is an atomic nucleus overlap. Its Fourier transform determines the distribution of the $N \bar{N}$-pair momentum relative to the residual nucleus.

For $P$-wave antiproton-nucleon interactions the relevant operator in momentum space is

$$
\widehat{a}_{1}=a_{1}\left(E_{B}-\frac{\boldsymbol{p}_{3}^{2}}{2 \mu_{12,3}}\right) \delta\left(\boldsymbol{p}_{3}-\boldsymbol{p}_{3}^{\prime}\right) \boldsymbol{k}_{12} \cdot \boldsymbol{k}^{\prime}{ }_{12}
$$


The steps from Eq. (C2) to Eq. (C7) may be repeated. As before the operator $\widehat{a}_{1}$ involves only internal coordinates in the $N \bar{N}$ subsystem and the average over recoil is given again by formulas similar to Eqs. $(\mathrm{C} 4),(\mathrm{C} 5)$ and $(\mathrm{C} 6)$. The overlap formula is more involved and in the coordinate representation one obtains (see the definition of $\gamma$ in Sec. II B)

$$
\left\langle\widehat{a}_{1}\right\rangle=\left.\left.\bar{a}_{1} \Theta \equiv \bar{a}_{1} \int d \boldsymbol{\rho} \boldsymbol{\partial}_{r}[\varphi(-\boldsymbol{r} / 2-\boldsymbol{\rho}) \psi(\gamma \boldsymbol{r}-\beta \boldsymbol{\rho})]\right|_{\boldsymbol{r}=0} \cdot \boldsymbol{\partial}_{r}\left[\varphi^{*}(-\boldsymbol{r} / 2-\boldsymbol{\rho}) \psi^{*}(\gamma \boldsymbol{r}-\beta \boldsymbol{\rho})\right]\right|_{\boldsymbol{r}=0} .
$$

The overlap may be calculated in spherical coordinates using radial and transversal derivatives $\partial_{r}, \partial_{T}$. For $S$ - wave nucleons only radial derivatives apply, for atomic wave functions one needs to calculate both derivatives. Mixed atomic-nuclear derivative contributes only to the radial term.

Atomic contribution obtained with $\psi(\boldsymbol{\rho})=N(n, L) Y_{L}^{m} \rho^{L} \exp (-\rho / n B)$ becomes

$$
\begin{gathered}
\Theta_{A}=\int \frac{d \boldsymbol{\rho}}{4 \pi}(\gamma)^{2}\left[\frac{L+1}{2 L+1}\left(\frac{1}{n B}\right)^{2}+\frac{L+1}{2 L+1}\left(\frac{2 L+1}{\rho}-\frac{1}{n B}\right)^{2}\right]\left[\frac{d \psi_{r}(\beta \rho)}{d \beta \rho}\right]^{2} \varphi_{r}(\boldsymbol{\rho})^{2} \\
\Theta_{N}=\int \frac{d \boldsymbol{\rho}}{4 \pi} \frac{1}{4}\left(\frac{d \varphi_{r}(\boldsymbol{\rho})}{d \rho}\right)^{2}
\end{gathered}
$$

where $\psi_{r}(\beta \rho)$ and $\varphi_{r}(\boldsymbol{\rho})$ are radial parts of the atomic wave functions, $\Theta \simeq \Theta_{A}+\Theta_{N}$ while the mixed term is small.

\section{Appendix D: Summation of multiple-scattering series}

The perturbation expansion for the level shift is, up to Coulomb corrections, equivalent to multiple scattering expansion. This follows from Trueman formula [see Eqs. (1) and (2)]. The method of summation used here is based on simple formula for meson scattering on two nucleons fixed at a distance $R$ obtained by Brueckner [35]. The scattering length on such a pair becomes

$$
A=\frac{2 a}{1+2 a / R}
$$

The term $2 a / R$ in the denominator sums the multiple scattering series. It has very simple interpretation, 2 is the number of scatterers, $a$ is the scattering amplitude on a single nucleon and $1 / R$ is the propagator for the meson bouncing off the fixed nucleons of the scattered particle. Expansion of the expression (D1) in terms of $2 a / R$ gives the multiple scattering series which is a geometric series. Equation (D1) is correct also for large $2 a / R$ when the multiple scattering expansion is divergent. This simple result indicates a possibility to sum the multiple scattering series by comparing it to a geometric series and correcting the sum at every step of the expansion. Let us present it as a series for the solution of a pseudo potential built as a multiple-scattering expansion with a potential given by Eq. (10). We denote single scattering term by

$$
\langle V\rangle=\left\langle\psi \varphi\left|\Sigma_{i} \frac{2 \pi}{\mu} a_{i}\right| \varphi \psi\right\rangle
$$

The double scattering term involves $G$ a propagator of the whole system in-between the subsequent collisions

$$
\langle V G V\rangle=\left\langle\psi \varphi\left|\left[\Sigma_{i} \frac{2 \pi}{\mu} a_{i}\right] G\left[\Sigma_{j} \frac{2 \pi}{\mu} a_{j}\right]\right| \varphi \psi\right\rangle
$$

and similarly for higher order terms. The first order sum is

$$
\langle V\rangle^{\prime}=\frac{\langle V\rangle}{1-\frac{\langle V G V\rangle}{\langle V\rangle}} .
$$

Expanding the denominator into a geometric series yields the first two terms of the multiple scattering. To have exact higher orders, one corrects the denominator. In this way one obtains a series in the denominator. Up to third order 


$$
\langle V\rangle^{\prime \prime}=\frac{\langle V\rangle}{1+C_{1}+C_{2}+C_{3}+. .}
$$

where the expansion coefficients are $C_{1}=-\langle V G V\rangle /\langle V\rangle, C_{2}=\langle V G V\rangle^{2}-\langle V G V G V\rangle /\langle V\rangle$ and

$$
C_{3}=\frac{\langle V G V\rangle^{3}}{\langle V\rangle^{3}}+\frac{2\langle V G V G V\rangle\langle V G V\rangle}{\langle V\rangle^{2}}-\frac{\langle V G V G V G V\rangle}{\langle V\rangle} .
$$

For illustration, we present the case of an $S$-wave projectile scattering on $N$ nucleons bound in $S$ waves in the nuclear c.m. system. The wave function is a product of Gaussian wave functions yielding a density radius mean square $R$. At zero projectile energy the propagator is taken to be

$$
G=-\frac{2 m}{4 \pi\left|r-r^{\prime}\right|}|\varphi\rangle\langle\varphi|
$$

where $m$ is the reduced mass of the projectile and of the nucleus, while $|\varphi\rangle$ is the nuclear wave function. The terms of expansion may be calculated in an analytic way, and the expansion parameter in this case becomes

$$
x=\frac{N a m}{R \mu}
$$

where $\mu$ is the reduced mass of the projectile and of a nucleon of the nucleus. One obtains $C_{1}=-0.977 x, C_{2}=$ $-0.045 x^{2}$ and $C_{3}=0.0076 x^{3}$. For light nuclei, like helium, the series converges rapidly. For the $2 P$ states, this convergence is much faster, the second order formula (D4) is sufficient for the present experimental precision.

Formula (D4) was checked against a full three-body calculation for low energy $\eta$-deuteron scattering [36]. It gives precision of a few $\%$ in a very demanding case of the virtual $S$ state close to threshold. As the deuteron is a loosely bound object one needs a better propagator allowing also for the deuteron excitation to continuum. Helium is a much stronger bound system and for the $2 P$ states such corrections are not necessary. What is necessary is a better description of the nuclear wave function $\varphi$. This is achieved here by the three-body model of interaction and the proper asymptotic form of the wave function (17). In this model the multiple scattering is a series of $V G V_{R}+\ldots$ where $V_{R}$ is the potential for the antiproton interaction with the residual system. For $S$ waves we take it as

$$
V_{R}=\frac{2 \pi}{\mu} \Sigma_{i}^{N-1} a_{i} \delta\left(\boldsymbol{r}_{\bar{p}}-\boldsymbol{r}_{i}\right)
$$

Thus, in this approach, the second order scattering term becomes

$$
\langle V G V\rangle=-\frac{2 m}{4 \pi}\left[\frac{2 \pi}{\mu}\right]^{2} \Sigma_{j}^{N} \Sigma_{i}^{N-1} a_{i} a_{j} \iint d \boldsymbol{r} d \boldsymbol{r}^{\prime} \psi\left(\boldsymbol{r}_{i}\right) \frac{\varphi\left(r_{i}\right) \varphi\left(r_{j}^{\prime}\right)^{*}}{\left|\boldsymbol{r}_{i}-\boldsymbol{r}_{j}^{\prime}\right|} \psi^{*}\left(\boldsymbol{r}_{j}^{\prime}\right)
$$

and similar formula is used for the $P$-wave interactions. We find the mixed sequence $S$-wave following $P$-wave scattering terms small. Hence, the summation via formula (D4) is used separately for $S S$ and $P$ P sequences.

Authors of Ref. [37. find the necessity of an additional term in the double scattering in $K D$ atoms because of $K^{-} \rightarrow K^{0}$ charge exchange. In the $1 S$ atomic state it has large effect. The related charge exchange process in antiproton atoms corresponds to a process initiated with the first collision of an antiproton on a proton which change into an antineutron and a neutron. In a second collision on a neutron the antineutron changes back to an antiproton. The link of events is

$$
(\bar{p} p) n \rightarrow(\bar{n} n) n \rightarrow n(p \bar{p})
$$

and corresponds to square of charge exchange amplitude for $\bar{p} p \rightarrow \bar{n} n$ given by isospin combination $T_{\text {ex }}=\left(T_{0}-T_{1}\right) / 2$. On the other hand $\bar{p} p \rightarrow \bar{p} p$ amplitude is given by $T_{\bar{p} p}=\left(T_{0}+T_{1}\right) / 2$. In the scattering region the charge exchange cross section is an order of magnitude smaller then total cross $\bar{p} p$ section and $\left|T_{e x} / T_{\bar{p} p}\right|^{2} \simeq 0.05$, see Ref. [7. One could expect the effect of the exchange process to be negligible but because of subthreshold $T_{0}$ dominance it turns to be very small, probably noticeable with improved potential models. One finds $\left|T_{e x} / T_{\bar{p} p}\right|^{2} \approx 0.15$ in the $S$ waves and $\approx 0.1$ in the $P$ waves for both Paris models. The real effect is additionally reduced by statistical factors from numbers 
of available $(p, n)$ pairs versus all available nucleon pairs. As the exchange term follows $\left(T_{0}-T_{1}\right)^{2}$ the correction enter as small enhancement or small reduction of the shifts and widths depending on positions of quasibound states. The calculated numbers have not been specified in the main text as the contributions are smaller then $1 \%$, much below the experimental precision. On the other hand, if the speculative Paris 09 suggestion of the $80 \mathrm{MeV}$ bound isospin 1 state turns out to be true, the strong cancellations of $T_{0}$ and $T_{1}$ amplitudes is removed in favour of $T_{1}$ dominance. The charge exchange scattering may then matter.

We are now in a position to check the convergence of expansion (D5) in the $2 P$ atomic state of Helium. Forgetting the small exchange term in the triple scattering one obtains, with the Gaussian density profile, $C_{1}=0.133 x$ and $C_{2}=8.92 \times 10^{-4} x^{2}$ with $x$ given by Eq. (D8) where $N=A-1$. The ratio $C_{2} / C_{1}=0.020 \mathrm{x}$. Thus $C_{2}$ terms contribute less than $1 \%$ to the total shifts and are neglected. On the other hand $C_{1}$ is to be calculated with the realistic nucleon wave function with the proper asymptotic behavior.

[1] J. Z. Bai et al. (BES Collaboration), Observation of a Near-Threshold Enhancement in the $p \bar{p}$ Mass Spectrum From Radiative $J / \psi \rightarrow \gamma p \bar{p}$ Decays, Phys. Rev. Lett. 91, 022001 (2003).

[2] M. Ablikim et al. (BESIII Collaboration), Spin-Parity Analysis of $p \bar{p}$ Threshold Structure in $J / \psi$ and $\psi(3686)$ Radiative Decays, Phys. Rev. Lett. 108, 112003 (2012).

[3] M. Ablikim et al. (BES Collaboration), Observation of a Resonance X(1835) in $J / \psi \rightarrow \gamma \pi^{+} \pi^{-} \eta^{\prime}$, Phys. Rev. Lett. 95, 262001 (2005).

[4] J.-P. Dedonder, B. Loiseau and S. Wycech, Photon or meson formation in $J / \psi$ decays into $p \bar{p}$, Phys. Rev. C 97, 065206 (2018).

[5] A. Sibirtsev, J. Haidenbauer, S. Krewald, Ulf- G. Meißner and A. W.Thomas, Near threshold enhancement of the $p \bar{p}$ mass spectrum in $J / \psi$ decay, Phys. Rev. D 71, 054010 (2005).

[6] B. El-Bennich, M. Lacombe, B. Loiseau and S. Wycech, Paris $N \bar{N}$ potential constrained by recent antiprotonic-atom data and $\bar{n} p$ total cross sections, Phys. Rev. C 79, 054001 (2009).

[7] J-P. Dedonder, B. Loiseau, B. El-Bennich, and S. Wycech, Structure of the X(1835) baryonium, Phys. Rev. C 80, 045207 (2009).

[8] D. Gotta, Review, Precision spectroscopy of light exotic atoms, Prog. in Particle and Nuclear Phys. 52, 133 (2004).

[9] D. Gotta et al., Balmer $\alpha$ transitions in antiprotonic hydrogen and deuterium, Nucl. Phys. A660, 283 (1999).

[10] M. Augsburger et al., Measurement of the strong interaction parameters in antiprotonic deuterium, Phys. Lett. B 461, 417 (1999).

[11] M. Schneider, R. Bacher, P. Blüm, D. Gotta, K. Heitlinger, W. Kunold, D. Rohmann, J. Egger, L. M. Simons, K. Elsener, X-rays from antiprotonic ${ }^{3} \mathrm{He}$ and ${ }^{4} \mathrm{He}$, Zeit. Phys. A 338, 217 (1991).

[12] PUMA, antiProton Unstable Matter Annihilation, CERN project, https://cds.cern.ch/record/2691045.

[13] A. Trzcińska et al., Information on anti-protonic atoms and the nuclear periphery from the PS209 experiment, Nucl. Phys. A692, 176 (2001).

[14] P. Lubiński, J. Jastrzębski, and A. Trzcińska, W. Kurcewicz, F. J. Hartmann, W. Schmid, and T. vonEgidy, R. Smolańczuk and S. Wycech, Composition of the nuclear periphery from antiproton absorption, Phys. Rev. C 57, 2962 (1997).

[15] B. El-Bennich, M. Lacombe, B. Loiseau, and R. Vinh Mau, Refining the inner core of the Paris $N \bar{N}$ potential, Phys. Rev. C 59, 2313 (1999).

[16] S. Deser, M.L. Goldberger, K. Baumann and W. Thirring, Energy Level Displacements in Pi-Mesonic Atoms, Phys. Rev. 96, 774(1954).

[17] T. L. Trueman, Energy level shifts in atomic states of strongly-interacting particles, Nucl. Phys. 26, 57 (1961).

[18] E.Lambert, Investigation of pionic atoms by means of scattering lengths. (in german), Helv. Phys. Acta 43, 713 (1970).

[19] J. Carbonell, J.M. Richard and S. Wycech, On the relation between protonium level shifts and nucleon-antinucleon scattering amplitudes, Zeit. Phys. A 343, 325 (1992).

[20] E.Klempt, C.Batty and J-M, Richard,The antinucleon-nucleon interaction at low energy: annihilation dynamics, Phys. Rep. 413, 197 (2005).

[21] J. S. McCarthy, I. Sick and R. R. Whitney, Electromagnetic structure of the helium isotopes, Phys. Rev. C 15, 1396 (1977).

[22] T.K. Lim, Normalization of the p- ${ }^{3} \mathrm{H}$ and $n-{ }^{3} \mathrm{He}$ tails of ${ }^{4} \mathrm{He}$ and the ${ }^{3} \mathrm{He}$ charge from factor, Phys. Lett. 44B, 341 (1973).

[23] M. P. Locher and H. J. Weber,Pion production in two-body pd collisions. Nucl. Phys. 876, 400 (1974).

[24] H.W. Fearing Distorted-wave impulse-approximation (p, $\pi$ ) calculations, Phys. Rev. C 16, 313 (1977).

[25] S. Wycech, A.M. Green anf J.A. Niskanen, On the energy levels in antiprotonic deuterium, Phys. Lett. 152B, 308 (1985).

[26] Jaroslava Hrtánková and Jiří Mareš, Antiproton nucleus quasi-bound states with the 2009 version of the Paris $\bar{N} N$ potential, EPJ Web Conf. 182, 01007 (2018).

[27] H. Pothet al., The antiprotonic x-ray spectrum of liquid helium, Phys. Lett. B 78, 523 (1977).

[28] R. Bizzari, Guidoni, F. Marcelia, F. Marcano, E. Castelli and M. Sessa, Antiproton-Deuteron Low-Energy Cross-Sections, Nuovo Cim. 22A, 225 (1974).

[29] T. E. Kalogeropoulos and G.S Tsanakos, Total and partial $\bar{p} d$ cross sections from 0.26 to 0.47 GeV/c , Phys. Rev. D 22, 2585 (1980). 
[30] F. Balestra et al., Annihilation of antiprotons at rest in ${ }^{3} \mathrm{He}$ and ${ }^{4} \mathrm{He}$, Nucl. Phys. A474, 651 (1987).

[31] E. Friedman and A. Gal, In medium nuclear interactions of low energy hadrons, Phys. Reports 452, 89 (2007).

[32] E. Friedman, A. Gal, B. Loiseau and S. Wycech, Antinucleon-nucleus interaction near threshold from the Paris $\bar{N} N$ potential, Nucl.Phys A943, 101 (2015).

[33] A. M. Green and S. Wycech, An optical potential for low-energy $\bar{p}$-nucleus interactions, Nucl. Phys. A377, 441 (1982).

[34] A. M. Green and S. Wycech, Widths of 4f antiprotonic levels in the oxygen region, Nucl. Phys. A467, 744 (1987).

[35] K.A. Brueckner, The Elastic Scattering of Pions in Deuterium, Phys. Rev. 90,715(1953).

[36] A. Deloff, $\eta \mathrm{d}$ and $\mathrm{K}^{-} \mathrm{d}$ zero-energy scattering: a Faddeev approach, Phys. Rev. C 61, 024004 (2000).

[37] S.S. Kamalov, E. Oset and A. Ramos, Chiral unitary approach to the $K^{-}$-deuteron scattering length, Nucl. Phys. A690, 494 (2001). 